\title{
Violencia, necropolítica y capitalocene en Cromo
}

Este ensayo explora la violencia espectacular presente en Cromo como mecanismo de acceso a una forma de violencia que somatiza los efectos de las prácticas capitalistas y examina la ciudad de Buenos Aires como espacio metafórico de las políticas económicas del mercado global en el territorio nacional. Partiendo de las ideas de Rob Nixon (violencia lenta) y Achille Mbembe (necropolítica) arguyo que, bajo el desamparo del Estado-nación, el ciudadano es despojado de su subjetividad y que el enfoque en los espacios permite explorar cómo el deseo de acumulación del capitalismo tardío afecta al individuo, la nación y el medio ambiente.

Palabras claves: serial televisivo, Argentina, violencia, necropolítica, neoliberalismo

This essay examines the presence of spectacular violence in Cromo as a point of entry into the effects of capitalist practices, and analyzes the role of Buenos Aires as a metaphoric space of the global marketplace within the political boundaries of the nation. Parting from theoretical ideas by Rob Nixon (slow violence) and Achille Mbembe (necropolitics) I argue that the disenfranchised citizen lives under the organizing logic of a politics of death. It is through the focus on the different spaces that the series demonstrates how the insatiable desire of late capitalist accumulation affects the individual, nation, and environment.

Keywords: television serial, Argentina, violence, necropolitics, neoliberalism

Threats from civilization are bringing about a kind of new "shadow kingdom," comparable to the realm of the gods and demons in antiquity, which is hidden behind visible world and threatens human life on their Earth. People no longer correspond today with spirits residing in things but find themselves exposed to "radiation," ingest "toxic levels," and are pursued into their very dreams by the anxiety of a "nuclear holocaust"... Dangerous, hostile substances lie concealed behind the harmless facades. 
Everything must be viewed with a double gaze, and can only be correctly understood and judged through this doubling. The world of the visible must be investigated, relativized with respect to a second reality, only existent in thought and concealed in the world. (Beck 72; énfasis mío)

Dirigida por los hermanos Lucía y Nicolás Puenzo - hijos del reconocido director, productor y guionista Luis Puenzo - y Pablo Fendrik, y estrenada el 6 de octubre de 2015 en horario central por la Televisión Pública argentina, la miniserie Cromo ha sido catalogada como un thriller que logra fusionar muy satisfactoriamente "elementos del discurso científico ... [y del] triángulo pasional ... [con] los inquietantes escenarios de la Antártida, la Patagonia y los esteros [argentinos] del Iberá" (Ferré). ${ }^{\mathrm{I}}$ La exitosa recepción de la serie a nivel nacional e internacional marca el triunfante debut de Lucía Puenzo - internacionalmente aclamada por la dirección de XXY (2007), El niño pez (2009) y Wakolda (2013) - en la pequeña pantalla. ${ }^{2}$ En una entrevista con los directores publicada en La Nación, Alejandro Lingenti señala que "[u]no de los objetivos de los responsables de Cromo es [su] exportación" - objetivo hoy en día satisfecho tal y como se desprende de la positiva recepción de la que ha disfrutado la miniserie tanto a nivel nacional como internacional. Galardonada con el "premio del concurso Popfcad para la Televisión Digital Abierta" (Bilbao), Cromo fue, en el 2015 , la única serie de Latinoamérica "seleccionada para la recién inaugurada sección de Series Prime Time del Festival de Cine de Toronto" (Ferré). La miniserie, que tiene como "visibles referentes" los seriales estadounidenses Twin Peaks (1990) de Mark Frost y David Lynch y True Detective (20I4) de Nic Pizzolato, ha sido elogiada también en "artículos del Screen Daily International y The Hollywood Reporter" (Lingenti) así como en otros "medios internacionales como Variety" (Ferré). Cabe señalar, además, que su disponibilidad en la plataforma digital Netflix así como la alta valoración de la serie por parte de sus espectadores se erigen como pruebas fehacientes de su exitosa recepción más allá de las fronteras nacionales.3

Desde una perspectiva crítica, Paul Julian Smith defiende la necesidad de estudiar los seriales televisivos al argüir que la televisión, "freed from cinema's lengthy lead time, is the first medium to engage with urgent contemporary social issues, working through them week by week for audiences in their millions" (I). Smith añade, además, que mientras que el asunto sociopolítico de las recientes series posee un valor intrínseco, "challenging viewers to abandon long-held prejudices and to confront rapid social change, these dramas ... are also notable for their asthetics" 
(2). Estos nuevos dramas "have high production values (easily the equal of cinema), intricately plotted narratives, and compellingly ambivalent characters" convirtiéndose, así, en artefactos culturales merecedores de un detallado análisis textual que hasta el momento no han recibido (2). José Valles Calatrava y Francisco Álamo Felices destacan, por su parte, las conexiones que el actual serial televisivo tiene con el folletín decimonónico arguyendo que "el gran interés narrativo del folletín - término hoy más dirigido al serial ... televisivo - ... , aparte de su estructura fragmentada y seriada, radica en su éxito de consumo, tanto en cuanto a gustos, por su funcionamiento conforme con los códigos ideológicos de la sensibilidad y percepción el mundo vigentes en su momento, como en lo referente a [su] amplia circulación mercantil" (cit. en Álamo Felices 75). Valles y Álamo añaden que "las series televisivas actuales, basadas en los mismos presupuestos compositivos tanto formales y articulares como de convencionalidad ideológica del folletín, alcanzan hoy las mismas cotas de éxito que aquel" (cit. en Álamo Felices 75). Cabe señalar, además, que un gran número de seriales televisivos se encuentran hoy en día disponibles en plataformas tales como Netflix, Hulu y Amazon lo cual hace posible que el espectador los consuma a demanda liberándose así, de las restricciones horarias impuestas por los canales televisivos. Esto ha dado lugar al fenómeno conocido como "binge-watching", esto es, el fenómeno que describe "any instance in which more than three episodes of an hourlong [sic] drama or six expisodes of a half-hour comedy are consumed at one sitting (L.A. Times cit. en Smecker). Para Frank Smecker, "[t]he act of binging ... implies the absence of any limit" siendo esta ausencia de límites lo que, según Smecker, caracteriza al capitalismo tardío ya que "late capitalism ... is distinguished by an unparalleled permissiveness, one that is taken to a hedonistic degree". El consumo de seriales televisivos responde, por consiguiente, a la búsqueda de placer que caracteriza a la contemporaneidad capitalista, una búsqueda "[that] serves to lower that unberable tensión we feel from working our ... jobs, day in and day out" evidenciando, así, la rehegemonización del formato folletinesco en el contexto del capitalismo tardío (Smecker).

La acción en Cromo gira en torno a la misteriosa muerte de Valentina (Emilia Attias), bióloga y reconocida profesora de la Universidad de Buenos Aires, que "muere en extrañas circunstancias mientras realiza una investigación en torno a una curtiembre en Corrientes" (Bilbao). Catalogada en un principio como accidental, la muerte de Valentina se torna, conforme avanzan los doce capítulos que componen la miniserie, "en el centro de una intriga policial y amorosa" que pone en riesgo la vida de Diego, su marido (Guillermo Pfenning), y Simón (Germán Palacios), 
amigo de ambos y amante de Valentina (Lingenti). La muerte de la bióloga sumerge al espectador en una especie de rompecabezas a través del cual "se va fragmentando lo que pasó" (Nicolás Puenzo, cit. en Lingenti). Por medio de la apelación afectiva que caracteriza el género de suspenso, la miniserie invita al espectador a participar del proceso de develación del misterio que engendra la trama. Es de destacar aquí, en línea con lo expuesto por Nicolás Puenzo - quien afirma que "[s]on los propios protagonistas, los dos científicos, los que van ensamblando las piezas para que la historia se vaya desarrollando" (cit. en Lingenti) - que las instituciones tradicionalmente encargadas de perseguir el crimen y castigar a los culpables ignoran su compromiso ético con la justicia relegando a los personajes a un estado de desamparo que les obliga a hacerse cargo de la investigación policial.4 Prueba de ello se observa en el capítulo 2, cuando Mirko (Alberto Ajaka) comenta que "la policía nunca se preocupó por el coche de Valentina" ni tampoco vino "por sus cosas de valor"; en el capítulo 4, cuando Simón le dice a Diego, "a la policía no le interesa lo que pasó con Valentina. Si no encontramos nosotros al asesino esto va a morir acá"; y en el capítulo 5, cuando Simón le reprocha al fiscal: " ¿No tiene tiempo para aceptar una denuncia de asesinato contra una curtiembre que es el sustento económico de un pueblo en donde la gente todos los días toma agua contaminada?". Lo que en un principio es concebido como objeto de la responsabilidad jurídica estatal acaba convirtiéndose en la responsabilidad individual mostrando así el proceso de desnacionalización de las obligaciones públicas característico de la contemporaneidad neoliberal y los daños colaterales que conlleva la persecución de la justicia al margen de la protección institucional.

Partiendo de estas ideas, las páginas a continuación tienen un doble propósito: por un lado, exploran la violencia espectacular presente en Cromo como mecanismo de acceso a una forma de violencia que, menos visible y paulatina en sus efectos, somatiza - ya sea en el cuerpo individual, social o medioambiental - los efectos de las ambiciosas prácticas capitalistas. Por otro, y en conexión con este primer objetivo, el trabajo aquí presente examina el papel de la ciudad de Buenos Aires - y más específicamente del lujoso apartamento de uno de los protagonistas, Víctor Anganuzzi (Daniel Veronese) - como espacio metafórico de las políticas económicas del mercado global en el territorio nacional, políticas que simultáneamente legitiman la constante expropiación territorial y la incesante explotación del ciudadano. Tomando como punto de partida los postulados teóricos elaborados por Rob Nixon y Achille Mbembe en torno a la violencia lenta (slow violence) y a la necropolítica, respectivamente, yo arguyo que bajo el desamparo del Estado-nación y sujeto a los designios 
del mercado global, el ciudadano vive gobernado por una forma de política en la que es despojado de su subjetividad bajo la lógica organizativa de la muerte. Es a partir de la presencia y el enfoque en los diferentes territorios donde transcurre la acción que la serie demuestra las múltiples maneras en que el insaciable deseo de acumulación que caracteriza al capitalismo tardío afecta al individuo, la nación y el medio ambiente.

VIOLENCIA Y ESPECTACULARIDAD

Si bien es cierto que Cromo ha sido clasificada como una miniserie perteneciente al "género policial científico" (Bilbao) e incluso al "ecothriller" (Hopewell), yo propongo su inclusión dentro del "neoliberal noir" - categoría genérica latinoamericana acuñada por Misha Kokotovic, quien señala que desde la década de los 90 del pasado siglo, "most of the continent has experienced something of a boom in narratives that use elements of detective or crime fiction to criticize the effects of the neoliberal, free market capitalism imposed on Latin American societies over the past ... decades" [while still maintaining] some degree of sympathy for the utopian projects of the Revolutionary Left" (15-6).5 A pesar de que el tono de denuncia hacia la corrupción que permea los diferentes organismos estatales está totalmente presente en la miniserie, esta muestra también un fuerte compromiso con la defensa del medio ambiente y con el reconocimiento de los sectores más oprimidos e invisibilizados de la sociedad evidenciando, de esta manera, un cierto vínculo con los proyectos utópicos de la izquierda latinoamericana.

Expectación, intriga, ansiedad e incertidumbre - ingredientes básicos del género de suspenso - se conjugan en Cromo desde la apertura del primer capítulo. La miniserie se abre por medio de una concatenación de tomas que, acompañadas de un sosegado toque de piano y el piar de los pájaros, invita al espectador a disfrutar de la sublime belleza contenida en los esteros argentinos del Iberá, situados entre las corrientes fluviales del río Paraná y Uruguay. ${ }^{6}$ La quietud del bello paraje natural y el templado acompañamiento musical son progresivamente interrumpidos por el lejano ruido de una lancha que gradualmente hace su aparición en escena. El espectador, posicionado en el interior de la embarcación segundos antes de que esta irrumpa la serena superficie acuática que impregna la pantalla, adopta - a medida que la barca aparece en escena - un punto de vista externo a la embarcación convirtiéndose en el único testigo de la actividad, aparentemente clandestina, que allí se está realizando. Acto seguido, la detención de la barca y el consecuente desvanecimiento del ruido del motor ceden paso a una música de ritmo más acelerado, con semblantes electrónicos, en la que predomina el uso de los instrumentos de viento 
acompañados por el toque de la percusión. Esto, en conexión con el uso de los guantes asépticos y los tubos de ensayo por parte de Valentina, la presencia de espuma sobre la superficie del agua, la aparición del cocodrilo y la enigmática toma de fotografías, subraya la tensión dramática en torno a la cual se va a desarrollar la acción. Cabe señalar aquí que esta primera escena finaliza, además, con el hallazgo de la protagonista por parte de lo que parece ser un guardabosques quien la sorprende y expulsa de lo que también aparenta ser una zona privada.

El limitado acceso a la información que posee el espectador llegado este punto se complementa, parcialmente, con los fragmentos de información contenidos en la subsiguiente secuencia de apertura. Entre ellos se encuentran: una fotografía, sumergida en el agua, en la que aparece la misma mujer que vimos a bordo de la lancha acompañada, ahora, por dos hombres; sangre; los cuerpos muertos de los mismos personajes retratados en la fotografía anterior anegados aquí entre la abundante vegetación que se oculta bajo las inmóviles aguas del estero; sangre; fragmentos corporales etiquetados en lo que parece ser una morgue; sangre; cuerpos muertos de peces hundidos en el agua; sangre; el cuerpo de una joven muchacha quien bucea en las profundidades de una transparente piscina y la entrada en escena de una barca que se funde con la aparente calma de la naturaleza, todo esto coreado por un intrigante acompañamiento musical y una entrecortada, al tiempo que sugestiva, respiración femenina.

La secuencia de apertura invita al espectador a ser consciente de la doble realidad sobre la que se va a desarrollar la acción a lo largo de la miniserie: la realidad de la superficie, la claramente visible, y la sumergida, aquella que con frecuencia es obviada y soslayada. Conviene señalar aquí, sin embargo, que la violencia espectacular - aquella encarnada en los difuntos cuerpos de los personajes - aparece en esta secuencia de apertura sumergida bajo la superficie del agua sugiriendo así, una intrínseca conexión entre lo visible y lo oculto. Llegado este punto quiero subrayar lo postulado por Ulrich Beck al comienzo del ensayo y argüir que Cromo hace que el espectador participe en el proceso de resolución del crimen incitándolo a investigar "the world of the visible ... with respect to a second reality ... concealed in the world" (72). Dicho de otro modo, la miniserie instiga al espectador a explorar la realidad que se esconde tras la fachada espectacular del acto de violencia que sirve como aparente eje de la trama central.

En su trabajo sobre la violencia, Slavoj Žižek establece una distinción entre lo que él denomina "subjective" y "objective violence" (I). La primera describe una forma de violencia física, inmediata y espectacular que es 
ejecutada por un agente claramente identificable (I). Esta forma de violencia constituye un acto perturbador del supuesto estado de normalidad de las cosas (2). La violencia objetiva, por el contrario, es aparentemente invisible e inherente al funcionamiento de las estructuras políticas y económicas que gobiernan la sociedad (I-2). Si bien la trama en Cromo gira en torno a lo que podría concebirse como un claro ejemplo de la violencia subjetiva - el asesinato de Valentina - lo que aquí me interesa es explorar otra forma de violencia que - inherente a las estructuras económicas del mercado global - es contenida, de forma menos visible, en el difunto cuerpo de la bióloga. Me refiero con ello a una forma de violencia referida, por Nixon, como "slow violence" ("Neoliberalism" 444). La "slow violence" - o violencia lenta o paulatina en español - es una forma de violencia "intimate and distant, unfolding over time and space on a variety of scales, from the cellular to the transnational, the corporeal to the global corporate" (444). A diferencia de la violencia espectacular, esta forma de violencia, que ocurre en la voz pasiva ("Slow Violence" 2), "no logra atraer con carácter de urgencia la atención política y social debido a la ausencia de su potencial visceral" (Caña Jiménez, "Mutantes, monstruos" 238). Sí logra, sin embargo, "somatizarse en aquello que Nixon denomina 'cellular dramas of mutation"' (cit. en Caña Jiménez 238) - efectos que aparentemente pasan inadvertidos pero generan "cambios fenomenológicos" (238). La violencia lenta somatiza, por consiguiente, los efectos nocivos que las prácticas capitalistas tienen no solo sobre el individuo y su posición dentro de la sociedad, sino también sobre el medio ambiente.

En relación con la importancia que el medio ambiente tiene en su producción televisiva, Lucía Puenzo afirma que uno de los objetivos de Cromo fue "correrse del costumbrismo y ... evitar el riesgo del pintoresquismo" (cit. en Lingenti). Si bien la plasmación de los hermosos escenarios naturales de Argentina y la Antártida ocupa un lugar central en el rodaje de cada uno de los capítulos y deleitan al espectador con su sublime belleza, la omnipresencia del medio ambiente va más allá de servir como escenario de la trama, al obligar al espectador a ser testigo del tremendo efecto que la actividad humana tiene sobre este. La omnipresencia del medio ambiente fuerza al espectador a repensar su relación con la naturaleza y a reconocer el estado de vulnerabilidad de grupos sociales cuyo derecho a la vida se encuentra, con frecuencia, comprometido en la vasta cadena de producción sobre la que se asienta el paradigma de la política económica neoliberal. Dicho de otro modo, la serie ofrece al espectador un punto de entrada para la exploración de las 
realidades menos visibles de lo que los científicos Paul Crutzen y Eugen Stoermer convinieron en llamar "anthropocene" (Royle 64).

El término "anthropocene" -antropoceno en español- fue acuñado en el año 2000 en un breve artículo publicado en el boletín informativo del "International Geosphere-Biosphere Programme" (Royle 64) y alude a una nueva época geológica que se encuentra definida por la actividad humana (63). El antropoceno es un concepto que encierra dentro de sí el reconocimiento del impacto negativo que la actividad del hombre tiene sobre el medio ambiente: el incremento de las emisiones de dióxido de carbono, la acumulación de los desechos plásticos, la vertiginosa deforestación de los bosques, la apresurada extinción de numerosas especies animales y el tan debatido cambio climático son solo algunas muestras de ello. Son numerosas las teorías en torno al momento histórico que marca el inicio de esta etapa geológica.7 $\mathrm{Si}$ bien hay lógica en todas y cada una de ellas, me inclino aquí a favorecer la fecha propuesta por Cruzen y Stoermer quienes ven en la invención de la máquina de vapor por parte de James Watt a finales del siglo XVIII, el origen de la Revolución Industrial $\mathrm{y}$, con él, el inicio de un nuevo modo de producción caracterizado por su impacto sobre el medio ambiente. Si bien, como señala Camilla Royle, "human societies [have always] involve[d] a relationship with the environment, capitalism differs from previous societies in its detrimental effects" (8o). Partiendo de las ideas de Ian Angus Royle añade, además, que "[t]he Anthropocene means an uncertain future, where human activity is in danger of pushing planetary conditions away from a 'safe operating space' for humanity towards a completely different type of world to which human societies may not even have time to adapt" (Royle 64).

Futuro incierto y espacio de operación seguro son palabras claves que problematizan la concreción y/o representación del impacto que la actividad humana tiene sobre el planeta en el aquí y ahora. Son numerosos los debates existentes en torno a asuntos tales como el acelerado cambio climático y/o la ineludible contaminación ambiental pero muy escaso el reconocimiento - ya sea político y/o cultural - de las comunidades humanas que, menos visibles y al margen del amparo social, están padeciendo a un nivel fenomenológico la frecuentemente obviada realidad del antropoceno. Conscientes de esta problemática, los hermanos Puenzo y Fendrik consiguen con éxito atraer la atención del espectador hacia los grupos más vulnerables posicionando en el centro de la acción a personajes pertenecientes a sectores de la población a menudo excluidos: me refiero con ello a sectores tales como el de los niños, el de las mujeres y el de los ancianos que habitan las zonas rurales. Para conseguir este 
objetivo, la miniserie otorga un rol activo al espectador quien, lejos de consumir de forma pasiva la ficción, es instigado a participar celosamente en el proceso de investigación. De la mano de Diego y Simón y a través del proceso de investigación por estos llevado a cabo, el espectador accede a las historias de unos personajes que podrían parecer anónimos. No obstante, y al igual que ocurre con la misteriosa muerte de Valentina, estas historias tampoco son presentadas en su totalidad desde el principio. No es hasta el capítulo 7 que el espectador consigue acceder - por medio del testimonio de Mirko, que es uno de los guardabosques de la curtiembre - a una narrativa íntegra que conecta, en un discurso organizado a partir de la lógica causa-efecto, fragmentos de historias identificadas y anónimas que hasta el momento habían sido presentados sin aparente orden ni concierto.

El capítulo 7 recoge la conversación mantenida entre Diego y Mirko, hombre de tez morena, pelo rizado, complexión fuerte y carácter curtido quien, por medio de la retrospección, relata "desde el principio" todo lo ocurrido con Valentina. ${ }^{8}$ Gracias al testimonio de Mirko, el espectador, al mismo tiempo que Diego, consigue acceder a la realidad que la curtiembre se ha esforzado en ocultar: la de la actividad clandestina de una empresa que obtiene el $50 \%$ de sus productos de cuero de la caza furtiva y que trata de forma clandestina el cuero tirando "los afluentes al río sin tratar" - de ahí los peces muertos flotando sobre la superficie del agua que aparecen en la toma anterior a la secuencia de apertura de este mismo capítulo. Mirko relata a Valentina y Simón - y a Diego y el espectador (ya que el espectador participa del proceso de develación del misterio de forma simultánea a Diego) - todo lo que queríamos saber: "que en e[se] pueblo, bebitos, nenitos, chiquititos, viejos ... son los primeros que sufren las consecuencias" de la contaminación del medio ambiente por desechos tóxicos, concretamente por la alta concentración de cromo en las zonas fluviales que irrigan el pueblo.9 "Son los más frágiles" - añade el guardabosques - y por eso "se enferman más rápido de cáncer [y] asma". Asma es la aflicción que Gema, hija de Mirko, comparte con un sinfín de personajes anónimos de la diegética provincia de Corrientes. Mirko comenta que la niña se enfermó cuando este trabajaba como cazador para la curtiembre, mostrando, así, los efectos indirectos y transgeneracionales que la violencia lenta postulada por Nixon tiene sobre cuerpos que, en apariencia, están desconectados del foco de la acción. El compungido padre confiesa que aunque sabía los motivos de la enfermedad de la niña "no pudo decir nada" porque él "estaba adentro", poniendo de manifiesto el miedo que mantiene al pueblo en un estado constante de sumisión con respecto a la curtiembre. El encuentro con Valentina supone, sin embargo, 
un punto de inflexión para el guardabosques quien le asegura a la bióloga que "las cosas van a cambiar" porque ella está ahí. Mirko muestra por medio de esta afirmación su complicidad con Valentina confiándole su ayuda en el proceso de recopilación de pruebas que les permitan "destapa[r] la realidad oculta" de la empresa y pone de manifiesto -en línea con lo teorizado por Beverley (y no podemos olvidar que Valentina es parte de la universidad y bióloga del CONICET) - "the ... desire not to be silenced or defeated, to impose [him]self on an institution of power and privilege from the position of the excluded, the marginal, the subaltern" (572). Es este compromiso ético entre Valentina y Mirko lo que desencadena la muerte de la bióloga ya que la curtiembre es un negocio grande que disfruta de impunidad en el que hay gente que no permite que haya otros que frenen su insaciable deseo de acumulación.

Por medio del relato de Mirko el espectador tiene acceso a los motivos que provocaron la violenta y repentina muerte de Valentina y a la igualmente violenta aunque paulatina afección que aqueja a los sectores más vulnerables de la región. Cabe destacar aquí el caso de Pedro Barrieta en el capítulo 3, niño de siete años de edad del que tenemos conocimiento, única y exclusivamente, gracias a la breve y fragmentada aparición de su historial médico en la pantalla. De él sabemos que tuvo que ser asistido con respiración mecánica pero que, aun así, falleció a causa de un paro cardíaco. Más limitada aun es la información en torno a Silvia Naso, a cuyo historial podemos acceder de forma muy breve para saber que es una mujer de 58 años a la que se le practicó una hematología. Los problemas de respiración compartidos por Gema y Pedro son también evidentes en Laura - guarda de seguridad de la curtiembre, hermana de Érika y madre soltera de dos pequeños niños.ro A diferencia de los casos anteriores, a Laura accedemos de manera directa, sin mediación del historial clínico o de lo relatado por una tercera persona. Laura aparece en escena tosiendo mientras se seca el pelo tras salir de la ducha después de regresar a casa de lo que parece haber sido un turno de noche en la curtiembre y es por medio del posterior testimonio de Mirko en el capítulo 7 que sabemos que aparte de su trabajo, Laura está también participando en la actividad clandestina llevada a cabo en la empresa. Esto explica el tono de reproche de Érika quien, habiéndose hecho cargo de sus sobrinos durante toda la noche, le dice a su hermana que su ropa apesta y que el motivo por el que esta no percibe el hedor es porque está inmersa en él durante todo el día. Es en el capítulo 7 también, y través del testimonio de Mirko, que el espectador conoce los motivos del "olor nauseabundo de ciertos horarios [y de] los desechos que tiraban al río cada vez que les avisaban que iba a caerles una inspección". Por medio de este breve comentario, el espectador 
se percata de que la empresa está exigiendo horas de trabajo extras a sus trabajadores quienes - directa o indirectamente - son obligados a doblar turnos para mantener en alza la producción del mercado negro.

La aparición de Laura en escena acto seguido a los historiales clínicos de Barrieta y Naso presagian, indudablemente, la muerte lenta del personaje. Al igual que Gema, Laura se erige como cuerpo en el que, de acuerdo a lo postulado por Nixon, se están engendrando "[the] cellular dramas of mutation" ("Neoliberalism" 445). Como ya apuntaba Mirko, son numerosos los casos de habitantes que, de manera anónima, son víctimas de la violencia lenta. El doctor Estévez comenta al respecto que: "[c]ada I5 o 20 días la clínica explota de pacientes, chicos descompuestos, ancianos que vienen con dermatitis severas, intoxicaciones" (capítulo 3). El anonimato de estas personas se yuxtapone en este mismo capítulo con el dramático acceso al difunto y bello cuerpo etiquetado con el nombre de Valentina Riera. El espectador accede a él de manera fragmentada a partir de lentos movimientos de la cámara y un grave acompañamiento musical que invitan al espectador a contemplar la espectacularidad de la violencia de la que ha sido víctima la bióloga. Si bien durante los primeros siete capítulos esta violencia espectacular -o subjetiva siguiendo los postulados de Žižek - parece erigirse como el motor de la acción, el testimonio de Mirko marca un giro en el desarrollo de la trama demostrando que la muerte de la bióloga no es más que el daño colateral de la violencia lenta teorizada por Nixon y, como consecuencia, una pieza más en la baraja de naipes a manos de la curtiembre. Valentina Riera es asesinada por recopilar pruebas sobre la actividad ilegal de la curtiembre y amenazar con destapar la impunidad de la que disfrutan sus altos cargos. Lejos de silenciar la realidad oculta de la curtiembre y poner fin al compromiso ético que la bióloga tiene con el medio ambiente y los sectores de la población más invisibilizados, la muerte de Valentina desvela la constante violencia perpetrada por la empresa y otorga reconocimiento a todas aquellas personas que, víctimas de una forma de violencia paulatina en sus efectos, pasan desapercibidas tanto para la clase política como para los altos mandatarios de la esfera económica.

Así, pues, y sirviéndose de la útil estructura dramática del género de suspenso, los hermanos Puenzo y Fendrik consiguen probar lo argumentado por Julie Sze: "that literature, arts, and the humanities offer a potential window into the lack of cultural recognition for the most oppressed and disenfranchised" dado que "art projects have the potential to highligh those places that are most unseen and unknown" (I03). Para Sze, el arte "facilitate[s] a politics of seeing that also expands cultural recognition by foregrounding the lives and experiences of those hardest hit 
by ecological injustice and those with the least responsibility for the problems" (IO3-04)." El reconocimiento de subjetividades afectadas por el antropoceno, exentas en gran medida de responsabilidad, me obliga a problematizar aquí las implicaciones éticas que conlleva el uso del término antropoceno para definir la etapa geológica marcada por la actividad humana. Si bien, como ya comenté, la definición del antropoceno contiene un cierto reconocimiento del impacto que la acción del hombre tiene sobre la naturaleza, hablar del ser humano de manera general conlleva la inclusión, dentro de una misma categoría, de sujetos con un mayor y menor grado de responsabilidad en los efectos que la actividad del ser humano tiene sobre el medio ambiente. Esto oculta, al mismo tiempo, la verdadera realidad de comunidades que, generalmente excluidas de recibir los beneficios económicos que genera la empresa capitalista, sí padecen e incluso somatizan los nocivos efectos que estas prácticas conllevan. Coincido aquí con Nixon quien señala que las narrativas sobre el antropoceno "[have generally] sidestepped the question of unequal human agency, unequal human impacts, and unequal human vulnerabilities" ("Great Acceleration") y con Sze quien añade que "the sidestepping of these perspectives of inequalities of agency or responsibility ... are not minor and irrelevant [but rather] reenac[t] and restag[e] violence, epistemological and enviromental" (IO5). Considero por ello más acertado el uso del término "Capitalocene" propuesto por críticos tales como Jason Moore como alternativa viable al antropoceno. Moore entiende el capitalismo como "a world-ecology [defined by] the accumulation of capital, the pursuit of power, and the co-production of nature in dialectical unity" (I). El término teorizado por Moore alude a una etapa de la historia "shaped by relations privileging the endless accumulation of capital" (5). "Capitalocene" es, por lo tanto, un término que, a diferencia del antropoceno, sí reconoce la desigual responsabilidad del hombre en el proceso de comodificación de la naturaleza probándose así lo ya extensamente argumentado por la crítica ecofeminista y postcolonial, "that not all humanity is equally responsable for either the carbon emissions that have led to anthropogenic climate change, nor the terraforming activities that have wreaked havoc on environments around the planet" (Anderson ix). Este reconocimiento testifica, a su vez, los igualmente desiguales efectos colaterales que conlleva la incesante comodificación del medio ambiente, apela a la identificación de los responsables y reta al individuo a repensar "the naturalized inequalities, alienation, and violence inscribed in modernity's strategic relations of power and production" (Anderson 2). Hablar de "Capitalocene" supone, entonces, reconocer la heterogeneidad de la humanidad y el inherente estado de violencia al que 
están sujetos amplios sectores de la sociedad como consecuencia del lugar que estos ocupan dentro de las alienantes estructuras sociales resultantes de las prácticas capitalistas.

VIOLENCIA, ESPACIO Y SOBERANÍA

Some lives are deemed more important than others. (Kelly I) El espacio e[s] ... la materia prima de la soberanía y de la violencia que acarrea.

(Mbembe 43)

En su estudio sobre la necropolítica, Mbembe hace eco de lo postulado por Franz Fannon quien arguye que "[l]a ciudad del colonizado es una ciudad agachada, una ciudad de rodillas, una ciudad revolcada en el fango" (cit. en Mbembe 30-3I) y añade que "la soberanía es la capacidad para definir quién tiene importancia y quién no la tiene, quién está desprovisto de valor y puede ser fácilmente sustituible y quién no" (Mbembe 45-46). Si bien lo teorizado por Fanon y Mbembe, respectivamente, alude a la espacialización de la ocupación colonial en sus diferentes períodos, este marco teórico constituye una base viable para entender las relaciones espaciales y soberanas que, presentes en Cromo, constituyen manifestaciones tangibles de la organización social que trae consigo el capitalismo tardío. Como bien señala Mbembe, en la actualidad:

impera una nueva concepción de la soberanía: la de aquellos actores internacionales que deciden quién debe vivir y quién debe morir en un momento dado, atendiendo a criterios estrictamente económicos ... las nuevas guerras ... son actos bélicos nomádicos que realizan empresas privadas ... que no buscan obtener territorio ni someter a las poblaciones; tan sólo afianzar recursos estratégicos y obtener beneficios inmediatos a cualquier coste. (contraportada)

Partiendo de la relación que el pulcro apartamento del empresario en la capital argentina mantiene con el contaminado y, en ocasiones, maloliente pueblo de Corrientes, exploraré en esta sección, la compleja maquinaria sobre la que se asienta el sistema de producción del capitalismo tardío - un sistema definido por la fragmentación corporal que caracteriza lo que François Guéry y Didier Deleule denominan "the productive body", el cuerpo productivo en español (59).12

Guéry y Deleule distinguen tres categorías de cuerpos: el cuerpo biológico, el cuerpo social y el cuerpo productivo y asocian cada uno de estos cuerpos con un período específico en el desarrollo del capitalismo 
histórico. El cuerpo biólogico es concebido como "the human apparatus, the linkage of vitality and physiognomy that cements birth, life, work, child-creation, physical decline, and death" (II). Este cuerpo corresponde a "the era of Handicrafts" - una etapa que abarca, en el continente europeo, desde finales del siglo XV a mediados del siglo XVI. ${ }^{13}$ El cuerpo social es aquel que emerge como resultado de la división del trabajo, donde diferentes individuos asumen diferentes tareas dentro una colectividad mayor (I2). Este cuerpo corresponde al periodo marcado por la industria de manufactura, desde la mitad del siglo XVI hasta el último tercio del siglo XVIII. Finalmente, el cuerpo productivo, que es el que aquí me interesa para entender las relaciones de poder que se desprenden de las prácticas económicas presentes en la miniserie, no es aquel que produce cosas en el sentido general de la palabra sino aquel que ha sido organizado "to produce commodities and thereby surplus-value, or profit, for the capitalist" (I2). El cuerpo productivo corresponde a la última etapa del capitalismo, esto es, la correspondiente a la industria a gran escala. Si bien el trabajador de la época artesanal era conocedor del proceso de producción del producto en su totalidad (2O), con la manufacturación tiene lugar la fragmentación de este conocimiento ya que "a laborer learns and executes only one part or moment of the production process, over and over again" (20). Esta fragmentación del conocimiento se intensifica mucho más durante el último período del capitalismo haciendo que la producción a gran escala sea testigo de la subordinación del individuo a la maquinaria industrial y del surgimiento "[of] a new stratum of observers ... entrusted with the knowledge of how to coordinate operations" (23). Estos observadores corresponden a los trabajadores no manuales y sirven de intermediarios entre los obreros y los capitalistas (23). El cuerpo productivo se caracteriza, por consiguiente, por la separación cuerpomente y la consecuente aparición de la figura del mediador quien, a cargo de organizar el conocimiento, "takes command of the work process by insisting on a virtual norm of ideal efficiency in all parts of the production process" (29). Este cuerpo productivo es imaginario no porque no exista sino, más bien, porque lo cognitivo "has been imagined as a separate force that can rule over the alienated worker" (29).

En línea con lo aquí expuesto, propongo examinar el diegético pueblo de Corrientes como metáfora del cuerpo productivo teorizado por Guéry y Deleule y el apartamento de Anganuzzi, en la capital argentina, como metáfora de la figura del mediador, esto es, como la fuerza cognitiva que, a partir de mecanismos de control y vigilancia, despliega su soberanía sobre la población que vive a expensas de la curtiembre. El apartamento de Anganuzzi media entre la dirección empresarial que tiene su sede en la 
desarrollada Suecia - a la que se accede en la ficción única y exclusivamente de manera indirecta por medio del uso que el empresario hace de dispositivos electrónicos tales como la computadora Mac y los teléfonos celulares - y la rural área de Corrientes dentro del territorio nacional. No debe obviarse aquí que la división campo y ciudad remite, en el contexto argentino, a la tradicional dicotomía de civilización y barbarie explorada por Domingo Faustino Sarmiento en Facundo (I845) quien asociaba los centros urbanos con los valores liberales, la modernidad y la civilización, y el campo con el atraso, el primitivismo y la barbarie. Cromo evidencia por el contrario - y en línea con lo postulado por Joanna Page en su extenso estudio sobre la crisis y el capitalismo en el cine argentino contemporáneo: "that the country can no longer be regarded as a counterpoint to the modernity of the city but it is simply the place where the contradictions of Argentine modernization and the inequalities of global capital are most clearly seen" (I2O). En línea con lo postulado por Page en relación con la gran pantalla, el campo en Cromo también se erige "as the sweatshop of urban development; as the corral of the hopeless and destitute; as the dumping ground for consumer-goods surplus" (I22)

Campo y ciudad - divisiones geográficas y sociales tradicionalmente yuxtapuestas - se renuevan en Cromo confirmando también lo postulado por Guéry y Deleule en su trabajo sobre el cuerpo productivo: que esta dicotomía pone de manifiesto la división entre los productores o trabajadores (los del campo) y los que no producen pero sí explotan (los de la ciudad) (68). ${ }^{4}$ El campo constituye, en este sentido, "the productive force whose production, overproduction, and sacrifices are consumed by the City" (70). Este sacrificio es materializado en la ficción a partir del proceso de somatización de la violencia lenta que genera el capitalismo tardío. Enfermedad, muerte y afección cristalizan así el sacrificio al que el cuerpo productivo -el pueblo de Corrientes- está sujeto bajo el poder soberano de la mente mediadora que, desde el lujoso apartamento en Buenos Aires, cataliza las políticas económicas y afectivas del mercado global en el territorio nacional.

Independientemente de si trabajan dentro o fuera del espacio físico que ocupa la curtiembre, todos y cada uno de los habitantes del pueblo desempeña una función dentro de su cadena de producción y funcionan como fragmentos enajenados del cuerpo productivo que es el pueblo en su totalidad. La única preocupación de cada habitante es desempeñar el trabajo asignado, ya sea este un trabajo manual, como el de los trabajadores que laboran dentro de la curtiembre, científico como el de Érika y/o jurídico, como el del fiscal y tantos otros personajes pertenecientes al cuerpo de policía. El pueblo es obligado a no conocer o - 
mejor dicho a fingir que no conoce - el total mecanismo de la empresa siendo esta la única forma de salvaguardar su única fuente de ingreso. Todo aquel que se opone, tal es el caso de Valentina, Diego y Nina (Malena Sánchez), acaba pagando su resistencia con la vida. La muerte - ya sea en su forma más espectacular o en su forma indirecta y paulatina - es, sin lugar a dudas, el principio organizador que rige la vida del pueblo - un pueblo que, en línea con lo postulado por Fanon al comienzo de esta sección - se caracteriza por su condición "agachada", "de rodillas" y "revolcada en el fango".

La horizontalidad conectada con el pueblo contrasta de forma visible con la verticalidad del lujoso apartamento de Anganuzzi en Buenos Aires. Residencia del CEO de la empresa a la que pertenece la curtiembre y padre de Nina - estudiante de biología y tesista de Valentina -, el ostentoso y moderno apartamento se eleva majestuosamente sobre la capital argentina y funciona como dispositivo de vigilancia y control del cuerpo productivo. Cabe señalar, en línea con esto, las amplias cristaleras que delimitan la vivienda que, si bien permiten la constante presencia de luz natural en el interior también hacen posible que Anganuzzi tenga acceso a toda la realidad que queda por debajo de su apartamento. Si bien desde el interior de la vivienda se puede acceder, sin obstáculo alguno, a todo lo que ocurre fuera, el interior es, por el contrario, un espacio de acceso privado: la elevación del mismo, entre otros muchos mecanismos de seguridad, hace imposible que el exterior - con la excepción de la luz solar y la mirada del espectador - pueda penetrar en él. Por su parte, la disposición elevada de la vivienda y el constante acto de vigilancia que en ella se despliega - no solo a partir de los amplios ventanales sino también a partir de mecanismos de control ejercidos por medio del constante uso de la tecnología (celulares, computadores y sistema de navegación GPS) remiten al espectador al panóptico teorizado por Michel Foucault y sirven como recordatorio del constante estado de vigilancia y escrutinio que también caracteriza a la contemporaneidad neoliberal. Cabe añadir, además, que a lo largo de casi toda la serie se escucha también una constante respiración superpuesta que, aparte de añadir suspenso a la trama, refuerza el estado de vigilancia y control bajo el que se encuentran los habitantes de Corrientes.

En línea con las prácticas de vigilancia, en el apartamento de Anganuzzi también se llevan a cabo prácticas de corte biopolítico conocidas como "technologies of responsibilization" - tecnologías que transfieren "[the] collective responsibility onto self-regulating individuals" (Cairns y Johnston 3). La biopolítica, tal y como la teorizó Foucault, "tiene que ver con la población, y esta como problema político, como problema a 
la vez científico y político, como problema biológico y de poder" ("Defender la sociedad" 222). La biopolítica hace referencia a un "conjunto de procesos que son propios de la vida [tales como] la mortalidad, [la] natalidad y [la] longevidad" (Ávila Fuenmayor y Ávila Montaño 3). Si bien el surgimiento de la biopolítica estuvo ligado a la Revolución Industrial y el consecuente interés por proteger la salud para prevenir la "disminución de la fuerza ... y del tiempo de trabajo [y el subsiguiente] aumento de los costos económicos" como consecuencia de "las enfermedades que atacan a una población en particular" (3), bajo el paradigma neoliberal tiene lugar la reescritura del concepto de biopolítica. Lo que originalmente estuvo conectado con la responsabilidad pública de las instituciones asistenciales pasa a convertirse, en la contemporaneidad política-económica, en la responsabilidad individual del sujeto. Prueba de ello se observa en Cromo a través del protagonismo que la piscina interior y la cinta caminadora tienen dentro del apartamento del empresario. Sendos artefactos remiten al espectador a prácticas tales como el "healthism" o "ideología del cuidado de la salud y el culto al cuerpo", poniendo de manifiesto la privatización de la salud pública - responsabilidad que antes recaía en las instituciones sociales (Caña Jiménez, "Neoliberalismo" I8). Cabe señalar, sin embargo, que no todos los sectores de la sociedad pueden participar de esta nueva forma de biopolítica ya que esta requiere un cierto poder adquisitivo. La privatización de la responsabilidad pública no es, por consiguiente, una práctica democrática al excluir de su disfrute a amplios sectores de la sociedad convirtiéndose, así, en un "criterio de distinción de clases" (Ortega I95).

Teniendo esto en cuenta, y en línea con lo teorizado por M.G.E. Kelly en torno a las diferentes formas del imperialismo biopolítico en la sociedad actual, arguyo que la biopolítica que gobierna la vida urbana materializada en el aséptico apartamento capitalino de Anganuzzi - es de carácter parasitaria ya que "death is figuratively exported and life imported back, in a systematic degradation of the possibilities for biopolitics in the periphery, arising out of the operation of biopolitics in the centre" (I8). La producción de excedente resultante de la productividad que genera el pueblo de Corrientes va ligada, ineludiblemente, a la violencia lenta somatizada en el cuerpo de cada uno de sus habitantes de modo que la muerte-en-vida de los personajes de Corrientes se convierte en la condición sine qua non para el ejercicio y el disfrute biopolíticos de la ciudad. El apartamento de Anganuzzi funciona, así, como metáfora espacial de una nueva forma de imperialismo que gobierna la contemporaneidad neoliberal. Me refiero a la necropolítica, entendida esta como una forma de "contrabiopoder ligado al concepto de necrocapitalismo ... capitalismo 
contemporáneo ... que organiza sus formas de acumulación de capital como un fin absoluto que prevalece por encima de cualquier otra lógica o metanarrativa" (Mbembe 15). En contraposición con la biopolítica que rige la vida del interior del apartamento como mediador de las políticaseconómicas del mercado global, el pueblo de Corrientes en su cualidad de cuerpo productivo se caracteriza por su condición de muerto-en-vida al estar sujeto a la soberanía -entendida esta como "el derecho de matar" (Mbembe 2I)- ejecutada desde el apartamento. Los hermanos Puenzo y Fendrik denuncian la lógica necropolítica de la contemporaneidad capitalista, evidenciando a su vez lo ya postulado por Marx, Deleule y Guéry, "that the productive body appears ... as a sick patient" (64), dado que el cuerpo productivo "in its guild form has been bled, corrupted, and tortured [and] [i]t has reached the completed form of large-scale industry only at the price of the degeneration of each of its vital forces" (65). Por medio de la serie, los hermanos Puenzo y Fendrik prueban también lo argumentado por Nixon en torno a la violencia lenta, que la globalización neoliberal "exacerbat[es] both uneven economic development and the uneven development of official memory" (46I). En relación con lo aquí apuntado en torno a la memoria oficial cabe señalar, llegado este punto, que el protagonismo que la serie otorga a los personajes marginales, el suicidio final de Anganuzzi en su piscina tras conocer la muerte de su hija y la revelación, ya sea directa o indirecta, de las confesiones y testimonios por parte de personajes tales como Valentina, Lena, Érika y Laura en el último capítulo ponen fin al silencio impuesto sobre el pueblo y remiten al espectador a los debates éticos en torno a la memoria que tanto interés han suscitado dentro de la producción cultural y crítica argentina de las últimas décadas. ${ }^{15}$ Así, pues, y por medio de Cromo, los hermanos Puenzo y Fendrik revisan - en la contemporaneidad neoliberal - la oficialidad de una Historia que ya condenada por el patriarca de la familia en su emblemático largometraje aún persiste en su esfuerzo por ocultar la realidad.

Virginia Tech

NOTAS

I Luis Puenzo es el director de La Historia oficial (1985), película argentina galardonada con múltiples premios tales como el Oscar a la mejor película extranjera en 1985. Puenzo es también el fundador de la productora argentina Historias Cinematográficas - empresa productora de la miniserie que aquí se 
analiza. Nicolás Puenzo es bien conocido por su trabajo de cinematografía en Bomba (2013) de Sergio Bizzio y Wakolda y su trabajo como productor de Infancia clandestina (20II) de Benjamín Ávila. Pablo Fendrick, por su parte, es el director de los largometrajes La sangre brota (2008) y El ardor (2014), protagonizada por Gael García Bernal y Alicia Braga.

2 Cromo fue creada "a partir de cuentos del periodista Martín Jauregui" que es a su vez el productor asociado de la serie y contó también con "el asesoramiento del biólogo Fernando Mejilde del Conicet [Consejo Nacional de Investigaciones Científicas y Técnicas de Argentina]" (Ferré).

3 En un artículo publicado para la página web \#borderperiodismo, Alejandra Casal comenta sobre el periodo de crecimiento "tanto de cantidad como de calidad" que están experimentando las series argentinas. "Ya sea por el talento actoral, creativo, de escritura y/o técnico" -añade- "las grandes cadenas y plataformas digitales (re)invierten en producciones argentinas". Casal apunta que el 2016 marca un antes y un después en cuanto al impacto que los seriales televisivos argentinos han tenido tanto a nivel nacional como internacional. Junto a Cromo, Casal también incorpora en su artículo series tales como El marginal de Luis Ortega, Estocolmo, identidad perdida de Nacho Viale y Diego Palacio y Proyecto vigilante de Andrés Rapoport y Alejandro Turner.

4 Como bien señala Asa Simon Mitman, "the genres of suspense, mystery, and horror derive their very names from the affects they are intended to promote - a sense of suspense, a sense of mystery, and a sense of horror" (6)

5 Aldo Ferrer distingue dos etapas en la construcción del Estado neoliberal en Argentina: la primera - señala - "tomó el atajo de la dictadura" ya que "el régimen fue impuesto por la fuerza, en el marco del terrorismo de Estado" (Iоo). La segunda etapa del neoliberalismo se sostuvo -defiende - "dentro de un régimen constitucional y fue ratificada por las urnas en los comicios del año I995" (IOI). La crisis argentina acaecida entre I998 y 2002 representa "la crisis del modelo neoliberal ... que intentó ... incrementar abusivamente el protagonismo de las relaciones de mercado, reduciendo a su vez el rol de los mecanismos estatales de control y planificación" (Galafassi). Cromo se estrena en el año 2015, año que pone fin a la etapa kirchnerista del gobierno argentino (2003-2015). Si bien el kirchnerismo se propuso reparar, tras la crisis, "un país dañado por la dictadura y por el neoliberalismo de los años noventa con políticas ... inclusivas de aquellos que habían sido expulsados de la economía y del bienestar" (Katz), el legado de esta etapa política no es otro que el cada vez más visible "incremento de la desigualdad ... y el retroceso de la educación ... la salud pública y ... la movilidad social" (Katz). Esto ha resultado en "un adelgazamiento constante de la vida civil" en Argentina ya que "grupos cada vez más numerosos de personas ha[n] ido perdiendo sus habilidades críticas para asumir los compromisos sociales y morales esenciales que se requieren 
para cumplir el papel de ciudadano en una democracia que funcione" (Katz). Esta falta de compromiso social y moral es, en principio, el denominador común de la población diegética del pueblo de Corrientes.

6 La secuencia de apertura de los doce capítulos que componen la miniserie aparece siempre precedida por una breve escena que, a modo de pieza suelta de un complejo rompecabezas, anticipa el asunto en torno al cual va a girar la acción del capítulo.

7 La fecha de inicio del antropoceno ha sido recientemente objeto de debate. Hay críticos que consideran que el periodo del antropoceno comenzó hace II.700 años - fecha generalmente asociada con el inicio del Holoceno que es el periodo en que tiene lugar la última edad de hielo. Es en este momento cuando las temperaturas relativamente cálidas en el hemisferio norte permitieron que las civilizaciones humanas se expandieran por el globo y desarrollaran la agricultura. Hay algunos que incluso sitúan el comienzo del antropoceno en un pasado más remoto, "when humans caused the extinction of many large mammals or even to the first surviving evidence of any human activity" (Royle 66). Estudiosos tales como Simon Lewis y Mark Maslin sugieren que el inicio del antropoceno coincide con el primer contacto entre los mundos después de I 492 -momento en el que "the two hemispheres were connected [and] trade became global" (175). Lewis y Maslin incluso señalan que hay científicos que se refieren a este momento "as the beginning of the modern 'world-system"'

(175). Otros han propuesto 1945 como el año de inicio de esta etapa ecológica por ser el año en el que tienen lugar las primeras experimentaciones con las armas nucleares (Royle 68). Stefffen et al. apuntan al respecto que "[t]he last 50 years have without doubt seen the most rapid transformation of the human relationship with the natural world in the history of the species. (258)

8 Mirko representa la voz del subalterno económico: su encuentro con Valentina y posteriormente con Diego y el espectador le brinda la oportunidad - en línea con lo postulado por John Beverley en torno al testimonio latinoamericano de traer su historia a la atención de la esfera pública (572). El testimonio de Mirko es complementado al final de la serie por el de otros muchos personajes.

9 Los desechos tóxicos no son el único objeto de la crítica medioambiental. También se critica, de manera mucho más sutil, el cambio climático que trae consigo el calentamiento global. Esto se observa en los apuntes en la pizarra "1965 $-4^{\circ} ; 2015-\mathrm{I}^{\circ}$. Colapso de Larsen" en la clase magistral dada por Diego en la Universidad.

Io Érika es bióloga en uno de los laboratorios pertenecientes al CONICET.

II La afirmación de Sze está contextualizada en torno al proyecto "Re-Locate Kivalina”. "Re-Locate Kivalina” es un proyecto artístico multidisciplinario elaborado en el 20I4 por académicos y artistas del California College of the Arts. Para más información véase su página web. 
I2 Las teorizaciones de Guéry y Deleule toman como base los postulados de Karl Marx presentes en el volumen I de Capital (1867).

I3 Esta etapa fue testigo "[of] the early moments of the transition into capitalism as a social formation that brought together the profits that flow from the break-up of feudalism, the dispossession of agrarian workers, long-distance mercantile trade, and usury" (Barnard y Shapiro I8).

I4 La productividad es entendida aquí como la capacidad de producir excedentes, de ahí la diferencia entre ser productor y ser productivo establecida por Guéry y Deleule.

I5 Los informes de Valentina revelados póstumamente confirman que "los valores de cromo en sangre humana eran letales y ... que el cromo encontrado en la sangre humana y el cromo utilizado por la curtiembre es el mismo". Por su parte Lena, Érika y Laura afirman, respectivamente, que "si la curtiembre hubiese tratado los afluentes antes de verterlos al río, [la población] no tendrí[a] es[os] niveles de cromo en sangre"; que "[l]os problemas de salud comenzaron a aparecer en muchos de los empleados de la curtiembre y habitantes del pueblo a principios [del año anterior cuando] empezó a funcionar el canal clandestino", y que "el señor Víctor Anganuzzi era el que tomaba todas las decisiones en la empresa ... [1] as legales y las ilegales". La imposición de silencio es acentuada a lo largo de toda la ficción a partir de la canción "Llevo un silencio a cuestas" de Guillermo Pessoa.

OBRAS CITADAS

ÁLAMO FELICES, FRANCISco. Los subgéneros novelescos: teoría y modalidades narrativas. Almería: U de Almería, 20 I.

A NDE RS ON, MARK. "Introduction: The Dimensions of Crisis". Ecological Crisis and Cultural Representation in Latin America: Ecocritical Perspectives on Art, Film, and Literature. Eds. Mark Anderson y Zélia M. Bora. New York: Lexington Books, 2016.

A NGU S, I A N. "Entering the Age of Humans". Socialist Review 4I3 (2016): S. pag. Web. Ávila fuenmayor, FRANCISCO y Claudia Ávila montaño. "El concepto de biopolítica en Michel Foucault". A Parte Rei 69 (2010): I-6.

BARNARD, PHILIP Y STEPHEN SHAPIRO. "Editor's Introduction to the English Translation". The Productive Body. Eds. François Guéry y Didier Deleule. Winchester: Zero Books, 20I4.

B ECK, ULR ICH. Risk Society: Towards a New Modernity. Trad. Mark Ritter. London: Sage, 1992.

beverley, john. "Testimonio, Subalternity and Narrative Authority”. A Companion to Latin American Literature and Culture. Ed. Sara Castro-Klaren. Malden:

Blackwell, 2008. 
B Il B A o, h o Ra ci o. “Lucía Puenzo: 'La serie es nuestro folletín moderno'”.

Clarín.com 6 de junio de 2015. S. pag. Web

CAIRNS, KATE y JOSÉE JOHNSTON. "Choosing Health: Embodied Neoliberalism,

Postfeminism, and the 'Do-Diet'". Theory and Society 4.2 (2015): 153-75.

CAÑA JIMÉNEZ, MARÍA DEL CARMEN. "Mutantes, monstruos y esperpentos: hacia una nueva concepción de la ciudadanía en la obra de Fernando Contreras Castro". Chasqui 45.2 (2016): 234-248.

—. "Neoliberalismo somático: sentimientos y afectos en Malos hábitos". Arizona Journal of Hispanic Cultural Studies 20 (2016): 9-28.

CASAL, A LEJANDRA. "Made in Argentina: la hora de las series nacionales". \#borderperiodismo.comi6 de septiembre de 20I6. S.pag. Web.

Cromo. Dir. Lucía y Nicolás Puenzo y Pablo Fendrik. Historias Cinematográficas. Netflix.

F E R É, L A U R A. "Lucía Puenzo estrena Cromo, un thriller de ciencia y pasión, por la pantalla de la Televisión Pública". Telam.com 2 de octubre de 2015. S.pag. Web.

FERRER, A L D O. "La construcción del Estado neoliberal en la Argentina". Revista de Trabajo 8.IO (20I2): 99-106.

F o u CA u t, M i h E L. "Defender la sociedad". Curso en el Collège de France (19751976). Trad. Horacio Pons. Buenos Aires: Fondo de Cultura Económica de Argentina, 2000.

G ALAFA SS I, G UID o. "Argentina: neoliberalismo, utilitarismo y crisis del EstadoNación capitalista”. Revista Herramienta 26 (2004): S. pag. Web.

GUÉRY, FRAN ÇO IS Y Didie R DELEULE. The Productive Body. Trad. Philip Barnard y Stephen Shapiro. Winchester: Zero Books, 2OI4.

h о Pe W L L, Jо н n. "Toronto: Pyramide, Puenzos, Fendrik Launch Cromo”. Variety Io de septiembre de 2015. S. pag. Web.

KATZ, A LEJA NDR o. "El neoliberalismo kirchnerista". La Nación 3I de enero de 20 I4. S. pag. Web.

K E L LY, M. G. E. Biopolitical Imperialism. Winchester: Zero Books, 2015. коко то Vic, міsна. "Neoliberal Noir: Contemporary Central American Crime Fiction as Social Criticism". Clues 24.3 (2006): 15-29.

LEWIS, SimON y MARK MASLin. "Defining the Anthropocene". Nature 519 (2015): I7I-I8O.

Lingenti, Alejandro. “Cromo, una serie federal”. La Nación 5 de octubre de 2015. S. pag. Web.

m B e m B E, A CH ll e. Necropolítica. Trad. Elisabeth Falomir Archambault. Madrid: Editorial Melusina, 20II.

MITTMAN, ASA SIMON. "Introduction: The Impact of Monsters and Monster Studies". The Ashgate Research Companion to Monsters and the Monstrous. Eds. Asa Simon Mittman y Peter Dendle. London: Ashgate, 20I2. I-I6. 
MOORE, JASON. "The Capitalocene. Part I: On the Nature and Origins of Our Ecoligical Crisis". Personal Website Jason W. Moore. 20I4. S. pag. Web. NIxо N, Rов. "Great Acceleration and the Great Divergence: Vulnerability in the Anthropocene". Profession is de marzo de 2014. S. pag. Web.

—. "Neoliberalism, Slow Violence, and the Environmental Picaresque". Modern Fiction Studies 55.3 (2009): 443-67.

-. "Slow Violence, Gender, and the Environmentalism of the Poor". Journal of Commonwealth and Postcolonial Studies I3.2-I4.I (2006-7): I4-37.

ORR, JU D IT H. Marxism and Women's Liberation. Winston-Salem: Bookmarks, 2015. O R T E G A, F RA N Cisco. El cuerpo incierto. Corporeidad, tecnologías médicas y cultura contemporánea. Madrid: Consejo Superior de Investigaciones Científicas, 20 Io. PAG E, Jo An na. Crisis and Capitalism in Contemporary Argentine Cinema. Durham: Duke UP, 2009.

ROYLE, CAM I la. "Marxism and the Anthropocene". International Socialism (2010): $63-82$.

SMECKER, FRAn K. "The Absolute Nothing of Binge-Watching: A Symptom of an Ascetic Ideal". Gadfly Online I6 de octubre de 2015. S. pag. Web.

Smith, Paul Julian. Dramatized Societies: Quality Television in Spain and Mexico. Liverpool: Liverpool UP, 2016.

St E F F N, Wil L E T A. Global Change and the Earth System: A Planet under Pressure. Berlin: Springer: 2004 .

SZE, JU LIE. "Environmental Justice Anthropocene Narratives: Sweet Art, Recognition, and Representation". Resilience: A Journal of the Environmental Humanities 2.2 (2015): IO3-II8.

Žı ž E K, SL A vo J. Violence: Six Sideways Reflections. New York: Profile Books Ltd., 2008. 